\title{
Modelo de sistema de locomoción autónomo: una ayuda para las personas con discapacidad visual en la prevención de la Covid-19
}

Autonomous locomotion system model: an aid to the visually impaired in prevention against Covid-19

DOI: https://doi.org/10.33262/rmc.v6i2.1248

\section{Elton Alvarenga Pessanha Junior ${ }^{1}$ \\ Instituto Federal Fluminense, Brasil \\ (iD) https://orcid.org/0000-0002-4084-8722. \\ elton.apj@gmail.com}

Simone Vasconcelos Silva ${ }^{2}$

Instituto Federal Fluminense, Brasil

(iD https://orcid.org/0000-0002-5994-6840. simonevs@iff.edu.br

\author{
Cristine Ferreira Nunes ${ }^{3}$ \\ Instituto Federal Fluminense, Brasil \\ iD https://orcid.org/0000-0003-1880-7681. \\ crisnfer@iff.edu.br
}

\section{RESUMEN}

Covid-19 generó una pandemia que afectó la vida de millones de personas en todo el mundo, sus sistemas de salud y economía, provocando la muerte de miles de personas. Según la Organización Mundial de la Salud, miles de millones de personas en el mundo tienen algún tipo de discapacidad visual y en Brasil, según el Instituto Brasileño de Geografía y Estadística, este número corresponde a millones de personas. En la situación de Covid-19, las personas con discapacidad visual son altamente vulnerables al riesgo de contagio debido a la necesidad de tocar superficies y dependen de la ayuda y la proximidad de terceros. Proponer un modelo de sistema de locomoción autónomo en ambientes exteriores e interiores para personas con discapacidad visual. El modelo propuesto se basa en dispositivos capaces de aportar mejoras en la calidad de vida y movilidad de las personas con discapacidad visual, permitiéndoles moverse de forma independiente en entornos exteriores e interiores, evitando así el contacto con superficies y terceros. Dividido en cinco pasos: (i) análisis de trabajos similares; (ii) modelo de 
detección de obstáculos y movimientos para asegurar la distancia; (iii) modelo utilizando Google Maps para la locomoción en entornos externos; (iv) modelo con la elaboración de mapas del entorno para la locomoción en interiores; (v) funcionalidades y ontología del sistema propuesto. El sistema propuesto puede componer un conjunto de medidas de protección contra el Covid-19 establecido por el Instituto Federal Fluminense, que puede ser utilizado por otras instituciones y gobiernos en apoyo de la Política Nacional de Movilidad.

PALABRAS CLAVE: Sistema autónomo; Deficientes visuales; Locomoción; Modelo.

\section{ABSTRACT}

Covid-19 generated a pandemic that affected the lives of millions of people around the world, their health systems and economy, causing the death of thousands of people. According to the World Health Organization, billions of people in the world have some type of visual impairment and in Brazil, according to the Brazilian Institute of Geography and Statistics, this number corresponds to millions of people. In the Covid-19 situation, the visually impaired are highly vulnerable to the risk of contagion due to the need to touch surfaces and depend on help and proximity to third parties. Goal. Propose a model for an autonomous locomotion system in outdoor and indoor environments for the visually impaired. The proposed model is based on devices capable of providing improvements in the quality of life and mobility of the visually impaired, allowing them to move independently in outdoor and indoor environments, thus avoiding contact with surfaces and third parties. Divide into five steps: (i) analysis of similar works; (ii) model for detecting obstacles and movements to ensure distance; (iii) model using Google Maps for locomotion outdoors; (iv) model with the elaboration of physical maps of the environment for locomotion indoors; (v) functionalities and ontology of the proposed system. The proposed system may comprise a set of protection measures against Covid19 established by the Fluminense Federal Institute, which may be used by other institutions and governments in support of the National Mobility Policy.

KEYWORDS: Autonomous system, Visually impaired, Locomotion, Model. 


\section{INTRODUCCIÓN}

Según datos del World Vision Report de la Organización Mundial de la Salud (OMS, 2019), se estima que 2.200 millones de personas en todo el mundo viven con algún tipo de discapacidad visual. En Brasil, según datos del Instituto Brasileño de Geografía y Estadística (IBGE, 2013), hay 6,5 millones de personas que tienen algún tipo de discapacidad visual (ceguera o baja visión) y en la mayoría de los casos estas personas tienen pocas condiciones financieras.

En enero de 2012 en Brasil entró en vigencia la Ley 12.587 que establece la Política Nacional de Movilidad y crea el Sistema Nacional de Movilidad Urbana. Cabe señalar que en el proceso de implementación de la movilidad urbana sostenible, la participación de todas las personas, incluidas las personas con discapacidad, es fundamental, ya que la movilidad y la capacidad de ir y venir son fundamentales para la identidad de las personas, sus experiencias y oportunidades de vida (Barbosa, 2016).

Las tareas rutinarias no son tan sencillas para quienes tienen limitaciones físicas, por ello, existe un gran esfuerzo por parte de investigadores de todo el mundo para crear innovaciones tecnológicas que puedan ayudar a las personas con discapacidad visual a desempeñarse autónomamente en las tareas cotidianas, así como en sus desarrollo social, educativo y profesional. Por lo tanto, el desarrollo de herramientas tecnológicas de bajo costo para personas con discapacidad visual se convierte en un gran desafío.

Según Campos (2020), en la época del Covid-19, una enfermedad provocada por el nuevo coronavirus, las personas con discapacidad visual deben tener un especial cuidado para evitar el contagio, ya que el contacto manual es su principal forma de interacción. Las formas en que se transmite la enfermedad, a través de gotitas de personas infectadas y al tocar superficies contaminadas, exponen a las personas con discapacidad a situaciones de riesgo. Según la Organización Nacional de Ciegos de Brasil, las situaciones cotidianas que viven las personas con discapacidad visual los hacen más vulnerables, ya que existe un uso frecuente de las manos en pasamanos, mesas, superficies, bancos y la necesidad de contacto directo con otras personas para ayudar con las actividades de la vida diaria, especialmente el apoyo de terceros a lo largo del camino en ambientes externos. Y la Asociación de Discapacitados Visuales del Estado de Río de Janeiro destaca que el principal desafío para los discapacitados visuales es la necesidad de usar el tacto todo el tiempo, estando más expuestos al contagio. 
En Brasil, el folleto informativo sobre los cuidados que deben tener las personas con discapacidad y enfermedades raras durante la nueva pandemia de coronavirus (Covid-19) publicado por el Ministerio de la Mujer, la Familia y los Derechos Humanos (MMFDH, 2020) solo ofrece algunas recomendaciones básicas para las personas con discapacidad visual, tales como: lavarse las manos con frecuencia y especialmente después de tocar mapas táctiles, pasamanos, manillas de puertas, entre otros, y usar gel de alcohol al 70\%; limpie los objetos que toque con frecuencia, incluido su bastón, con agua y jabón y alcohol al 70\%; al recibir ayuda sosténgase del hombro, evitando tocar las manos o el codo de la persona que lo guiará, ya que la recomendación es que al toser o estornudar la gente lo haga en la mitad del brazo; y la descripción de la forma correcta de lavarse las manos. Así, el gobierno brasileño solo propone recomendaciones simples y no presenta una política de prevención para las personas con mayor vulnerabilidad al contagio.

En Brasil, las personas con discapacidad visual enfrentan importantes desafíos diarios, uno de los mayores desafíos es la locomoción segura y la mayoría de las personas con discapacidad necesitan la ayuda de otros para moverse con éxito. Con la pandemia, esta situación se volvió aún más crítica. Aunque con el desarrollo tecnológico han surgido algunas herramientas electrónicas que ayudan en las trayectorias diarias, herramientas como un perro guía y un bastón siguen siendo las más utilizadas. Mudarse en interiores también es un gran desafío a superar por personas con discapacidad visual, muchas instituciones no cuentan con centros de apoyo para estas personas, tareas cotidianas imprescindibles como ir al baño, cafetería y biblioteca se vuelven inviables sin el apoyo de terceros o núcleos especializados.

Vasconcelos et al. (2020) realizó una investigación bibliográfica sobre tecnologías asistenciales (TAs) para personas con discapacidad visual, donde se analizaron veinte artículos. A partir de este análisis, los autores concluyeron que: (i) el uso de aplicaciones como los TAs es más exitoso que el uso de otras tecnologías, ya que permite la orientación espacial, la detección de obstáculos y es capaz de orientar a los usuarios en las rutas y rutas deseadas; (ii) los TAs gestionados por teléfonos inteligentes, tabletas $\mathrm{y}$ computadoras lograron reducir el tiempo de viaje hasta en un 50\%, reduciendo los espacios entre los puntos de salida y llegada, y la dificultad de la orientación espacial; (iii) el uso de la tecnología permite a las personas con discapacidad visual tener mayor libertad y autonomía para elegir los caminos que desean tomar; (iv) el uso integrado de la misma tecnología para áreas internas y externas aún presenta dificultades, por lo que 
es necesario intensificar los estudios en este aspecto; (v) la mayor dificultad para integrar la tecnología para dar servicio a las áreas exteriores e interiores es la imposibilidad de que la señal GPS (sistema de posicionamiento global) y GIS (sistema de información geográfica) satisfaga toda la diversidad de entornos frecuentados por los usuarios y la complejidad de hacer que todos los entornos estén conectados de manera viable.

En este contexto, el objetivo de este trabajo es proponer un modelo de sistema autónomo de locomoción en entornos externos e internos para personas con discapacidad visual. El modelo propuesto se basa en un conjunto de dispositivos (microcontrolador, sensores ultrasónicos, teléfono celular con sistema Android y software), pudiendo aportar mejoras en la calidad de vida y movilidad de las personas con discapacidad visual, permitiéndoles moverse externa e internamente en una de manera adecuada y independiente, evitando así el contacto con superficies y terceros, reduciendo el riesgo de contagio por Covid-19.

A partir de la introducción, este trabajo se divide en las siguientes secciones: Sección 2 discute algunos trabajos relacionados con el tema abordado, Sección 3 - presenta la metodología propuesta, Sección 4 - trae el modelo de sistema de locomoción autónomo propuesto en este trabajo y la Sección 5 aborda las conclusiones.

\section{TRABAJOS RELACIONADOS}

Los trabajos relacionados abordan el uso de aplicaciones para proporcionar tecnologías de asistencia para personas con discapacidad visual. Estos trabajos se dividieron en tres categorías según la aplicabilidad de la tecnología de asistencia: entorno interno, entorno externo y entorno interno/externo.

\section{Entorno Interno}

Li et al. (2019) presentan un sistema de navegación asistida móvil para ayudar a personas con discapacidad visual a través de la orientación y detección de obstáculos, utilizando un teléfono inteligente con cámara para detectar movimiento y un bastón para vibración, conectado a la aplicación. Como resultado, obtuvo una reducción en el tiempo de viaje, una mejor orientación espacial y detección de obstáculos.

Huang et al. (2018), propone el desarrollo de un proyecto mediante la lectura del diseño del entorno para orientar a las personas con discapacidad visual, con la ayuda de una computadora. Como resultado, el trabajo generó el diseño del entorno y los participantes 
llegaron a su destino con una distancia menor, pero no se analizó el cumplimiento de las rutas.

Cheragui et al. (2017) evaluaron un sistema de guía de localización en interiores utilizando balizas desplegadas en interiores. Utilizaron un teléfono inteligente, mapas interiores y un sistema de altavoces bluetooth. Los autores concluyeron que una limitación del sistema es la necesidad de mejorarlo según las preferencias y características del usuario.

Rafian y Legge (2017), analizaron si a partir de fotografías tomadas por personas con discapacidad visual es posible generar estimaciones precisas de su localización interna, a través de la asistencia humana remota y el crowdsourcing. Los recursos utilizados fueron el teléfono inteligente, el plano de la planta y las imágenes representativas. El sistema propuesto fue efectivo, sin embargo, necesita personas sin discapacidades para ayudar.

Tao et al. (2017), validó el uso de herramientas para instrucciones de navegación para personas con discapacidad visual a través de un programa de prueba, donde es posible guiar a un avatar por un camino determinado. Se utilizaron sensores de radiofrecuencia y altavoz para teléfonos inteligentes. El sistema identificó todos los obstáculos, generó rutas y proporcionó orientación espacial. A pesar de estos resultados, la evaluación fue solo digital, por lo que fue necesaria una prueba práctica.

Zhang y Ye (2016) evaluó la tecnología de asistencia "Pose Estimation" para localizar al usuario en un plano de planta y guiarlo a un destino determinado, utilizando un bastón, sensores de obstáculos y un sistema de altavoces Bluetooth. Todos los experimentos fueron exitosos, el sistema se consideró fácil de operar, sin embargo, funciona mejor en área externa.

Flores et al. (2014), rastreó el camino recorrido (guía y detección de obstáculos), construyendo un camino seguro de regreso al punto de partida, mediante el uso de sensor de ultrasonido, sistema de información geoespacial (GIS), sistema de posicionamiento global (GPS) y vibración para la comunicación. El sistema fue capaz de detectar el 85\% de los obstáculos, reduciendo el tiempo de navegación y guiando con precisión.

Jaind et al. (2013), implementó un sistema de navegación interno portátil y autónomo, el cual fue implementado en un edificio universitario. Para ello, utilizaron los siguientes recursos: teléfono inteligente, sistema de detección de velocidad de calzado y sistema de altavoces Bluetooth. El sistema se consideró eficaz, ya que se redujo el ruta y el tiempo. 
Legge y col. (2013), implementó un sistema de localización espacial que permite a las personas con discapacidad visual moverse, identificando obstáculos y orientándose espacialmente. Utilizaron una cámara RGB-D, micrófono, computadora portátil, sistema Bluetooth para altavoz y base de datos. El sistema se consideró eficiente e independiente, pero algunos de sus equipos no son de fácil acceso.

Manduchi (2012) evaluó cómo las personas con discapacidad visual interactúan con un sistema de visión móvil para el descubrimiento de rutas y tareas de orientación, a través de teléfonos inteligentes, auriculares y mapa interno. El sistema tuvo un resultado positivo, ya que redujo la duración del curso y, en consecuencia, el tiempo.

Manduchi et al. (2010) evaluaron un sistema que utiliza marcadores de colores especiales, colocados en lugares clave del entorno, que pueden ser detectados por un teléfono con cámara normal. Utilizaron un lector de sensor digital, sistema Bluetooth para altavoz y teléfono inteligente. El sistema pudo identificar los obstáculos y las coordenadas correctas. Sin embargo, es necesario integrar el sistema para obtener una mayor practicidad.

\section{Entorno Externo}

Barati y Delava (2015), proponen un sistema con el objetivo de guiar a las personas con discapacidad visual para que reconozcan los obstáculos a través de la implementación de un sistema de sensores móviles de navegación y orientación controlados por computadora. El sistema demostró ser efectivo para la orientación espacial del usuario, pero no pudo guiar y detectar obstáculos de manera efectiva.

Jafri y Ali (2014), desarrolló una aplicación para personas con discapacidad visual, basada en un sistema de posicionamiento global. Esta aplicación permite a los usuarios registrar rutas de uso frecuente, así como rutas privadas deseadas por el usuario. Además de emitir señales de alerta en situaciones de riesgo y registrar los obstáculos que se encuentren en su camino.

Abudullah et al. (2014), creó un prototipo para desarrollar la movilidad urbana para personas con discapacidad visual en un sistema de transporte público, específicamente para el transporte en autobús. El prototipo dispone de módulo de detección de obstáculos, identificación de la localización de la parada y número de identificación de su ruta. El objetivo principal del proyecto era incrementar la independencia del usuario en su locomoción diaria en el transporte público. 
Loeliger y Stockman (2013) evaluaron un sistema de mapas de audio interactivo para personas con discapacidad visual, con el objetivo de guiar, orientar y detectar obstáculos. Utilizaron marcadores de color, un teléfono inteligente para lectura de marcadores y un sistema de altavoces Bluetooth. Como resultado, observaron que el tiempo caminado disminuyó considerablemente y que los participantes de la prueba reportaron una mayor confianza al caminar.

\section{Entorno Externo y Interno}

En el proyecto desarrollado por Castilho-Cara et al. (2016), como se muestra en la Figura 1, se utilizaron módulos Bluetooth conectados a un microcontrolador para recibir señales, placas Bluetooth en puntos estratégicos en un ambiente cerrado (edificios, oficinas y escuelas) para emitir señales. La señal de Bluetooth en cada punto estratégico indica direcciones y coordenadas para que las personas con discapacidad visual se muevan sin la ayuda de un bastón. Pero uno de los grandes problemas es la gestión de la batería, ya que este sistema no funciona durante periodos prolongados. Para la locomoción en ambientes abiertos, se implementó este sistema en señales de tránsito, informando al usuario del estado de color de los semáforos de la ciudad.

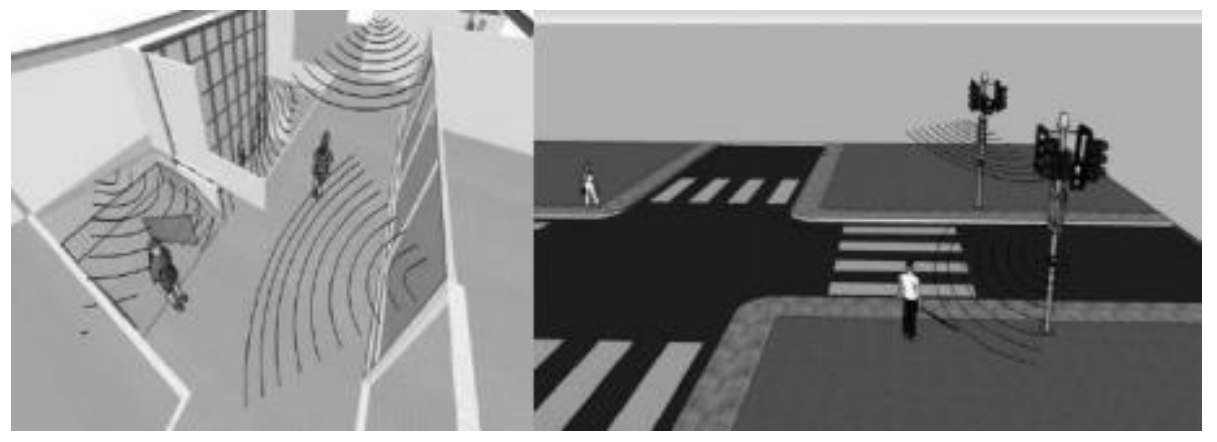

Figura 1. Entorno interno y externo.

Fuente: Castilho-Cara et al., 2016.

Valentim et al. (2016) presentan un bastón electrónico inteligente para la movilidad de personas con discapacidad visual y tiene capacidades como reconocer personas, objetos e identificar obstáculos a la altura de la cintura y la cabeza, así como desnivel como agujeros y escalones. La identificación de obstáculos se realiza a través de sensores ultrasónicos ubicados en varias partes como: puño de bastón, sombreros o collar y en el extremo inferior. Desde la conexión del celular, el usuario recibe señales de alerta a través 
de comandos de voz previamente grabados o señales sonoras personalizadas para informar el tipo de riesgo identificado, que puede ser obstáculo o desnivel y la distancia respectiva.

Barati y Delavar (2015) desarrollaron un prototipo para la movilidad de personas con discapacidad visual a entornos abiertos y cerrados utilizando sensores ultrasónicos y un módulo GPS. En este estudio, es posible almacenar los obstáculos que se detectan en el camino en una base de datos del sistema. Cuando se detectan obstáculos, el prototipo emite vibraciones en respuesta, por lo que la persona discapacitada es consciente de que los obstáculos están separados por 400 centímetros. Este sistema constaba de un módulo de reconocimiento de localización, un sistema de posicionamiento global para diseñar rutas, una base de datos para almacenar información, una interfaz de usuario, un microcontrolador, un sensor ultrasónico, un módulo bluetooth y un vibrador.

Wang et al. (2014) proponen una ayuda computacional para la navegación, verificando la efectividad de los marcadores de color mediante el uso de estos marcadores, lectores de marcadores y un sistema de altavoces Bluetooth. El sistema reconoce la localización pero no proporciona direcciones de conducción.

Koutny y Miesenberger (2015) presentaron un prototipo que utilizaba una tecnología denominada entrenador de movilidad virtual, esta tecnología fue desarrollada a partir de un entrenador humano sin discapacidad visual que proporcionó información para desarrollar rutas previamente probadas por él. Este fue el primer trabajo que permitió crear rutas con la ayuda de humanos capacitando a discapacitados para una mejor movilidad.

Coughlan y Manduchi (2009) validaron la efectividad de los marcadores de color para etiquetar ubicaciones específicas e investigaron diferentes estrategias de búsqueda para la detección de marcadores. Los recursos utilizados fueron marcadores, cámara de teléfono inteligente y sistema de altavoces Bluetooth. El sistema no fue efectivo, ya que solo el $33 \%$ de la muestra completó el experimento.

\section{METODOLOGÍA}

La metodología propuesta en este trabajo se divide en cinco etapas, donde las Etapas II a V componen el modelo para el sistema autónomo, como se muestra en la Figura 2:

- Etapa I - Análisis de soluciones adoptadas en trabajos similares; 
- Etapa II - Propuesta del modelo de detección de obstáculos y detección de movimientos para asegurar la distancia. Esta es la parte hardware, que incluye el bastón con sensores ultrasónicos y ópticos conectados a un microcontrolador que se comunica con el celular; - Etapa III - Propuesta del modelo para entorno externo, parte software, que incluye la recolección de datos de Google Maps, comunicación GPS (Google Maps) con el celular y la aplicación, para locomoción en áreas abiertas;

- Etapa IV - Propuesta de modelo para entorno interno, parte software, que incluye la elaboración de mapas computerizados específicos del entorno, lectura y audio para locomoción en interiores;

- Etapa V - Funcionalidades y ontología del sistema propuesto.

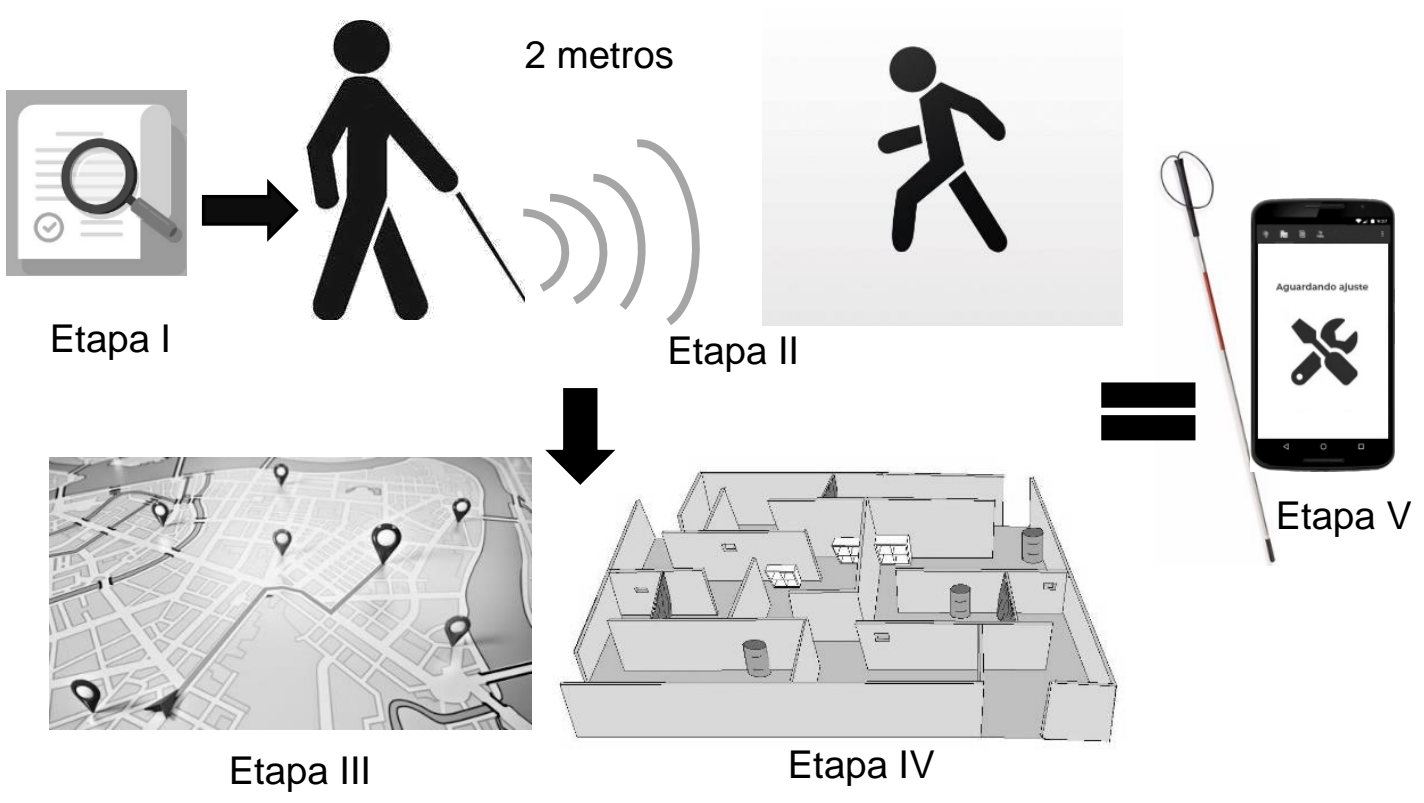

Figura 2. Etapas de la metodología propuesta.

\section{RESULTADO - MODELO DE SISTEMA AUTÓNOMO}

A través de la investigación bibliográfica, se diseñó un conjunto de alternativas para resolver el problema de movilidad de las personas con discapacidad visual. El modelo propuesto que permite la locomoción tanto en calles, avenidas y plazas se denomina locomoción en entornos externos. El modelo que permite la locomoción en edificios, centros comerciales, bancos y otros establecimientos se denomina entornos internos.

El proyecto tiene como objetivo integrar tecnologías existentes y a partir de ellas desarrollar una tecnología innovadora que sea eficiente y de bajo costo, destacando que 
con solo un Smartphone que contenga un paquete de datos / internet y un bastón electrónico (sensores), las personas con discapacidad visual podrán moverse de forma independiente.

El modelo propuesto se divide en cuatro etapas, la primera etapa está relacionada con el hardware, construcción del bastón electrónico, y las otras con el software, desarrollo de aplicaciones.

\section{Modelo de bastón}

El modelo propone el diseño de un bastón electrónico que contiene sensores para detectar obstáculos y movimiento. El bastón tiene sensores ultrasónicos y ópticos conectados a un microcontrolador que se comunica con el celular.

La detección de obstáculos capta los objetos que se encuentran distantes y frente a la persona discapacitada, formando un semicírculo desde la cintura hasta el suelo y desde la cintura hasta la parte superior da la cabeza, evitando accidentes durante el trayecto.

La detección de movimiento se refiere a capturar los movimientos en un radio de 2 metros (medida recomendada por las agencias de salud para mantener la distancia necesaria para evitar el contagio del Covid-19) de los discapacitados que se encuentran hacia el centro del cuerpo de los discapacitados.

Al detectar el obstáculo, el bastón emitirá un bip (señal sonoro) con un aumento gradual del audio a medida que la persona discapacitada se acerque al obstáculo. Al detectar el cuerpo en movimiento, el bastón emitirá pequeñas vibraciones que aumentan de intensidad a medida que el cuerpo se acerca.

Para la elaboración de este bastón se necesitará el siguiente equipamiento: microcontrolador, sensor ultrasónico, sensor óptico difuso y bastón.

\section{Modelo de locomoción en entorno interno}

El modelo propone que las personas con discapacidad visual se muevan con mayor seguridad y confianza utilizando el bastón electrónico en interiores.

El modelo trabaja con mapas específicos de entornos cerrados construidos y añadidos a la aplicación, como se muestra en la Figura 3. Así, se propone que la aplicación almacene y lea los mapas físicos específicos de cualquier tipo de establecimiento, facilitando el movimiento de la persona discapacitada. 


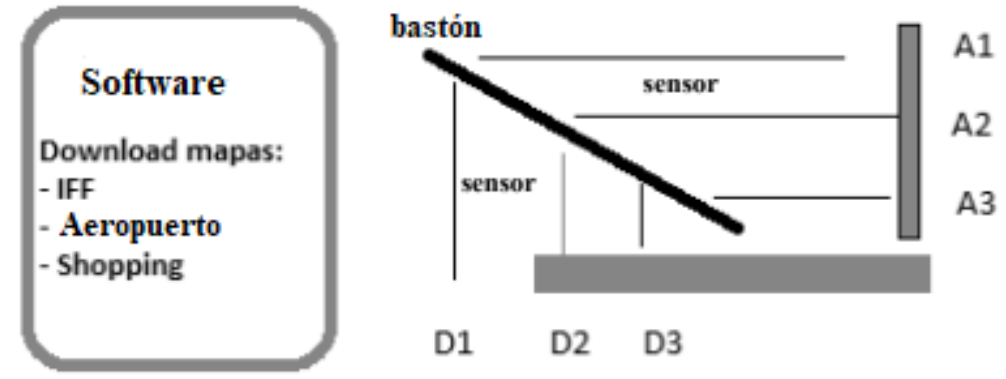

Figura 3. Bastón electrónico y aplicación para entorno interno.

Tanto para el modelo de locomoción en interior como en exterior, se utilizará lo bastón electrónico con la ayuda de sensores ultrasónicos, acelerómetro y microcontrolador, donde los sensores servirán para medir la distancia de cuerpos, objetos u obstáculos y como señal de salida el microcontrolador enviar una respuesta sonora y/o vibratoria. Los obstáculos se clasificarán en categorías (altura A / profundidad D) y se pueden registrar en el mapa de ruta generado.

\section{Modelo de locomoción en entorno externo}

El modelo propone el uso de sistemas de posicionamiento global/Google Maps, permitiendo al usuario registrar sus propias rutas y obstáculos con la ayuda de un bastón electrónico, mediante el uso de sensores, como se muestra en la Figura 4.

En este modelo, se propone que la aplicación, utilizando mapas de Google Maps, defina la orientación de la persona discapacitada, que también puede crear sus propias rutas preferidas. En este caso, el GPS conectado a la red de datos del celular descargará los mapas y al descargar el mapa de su ruta, el usuario podrá realizar la ruta.
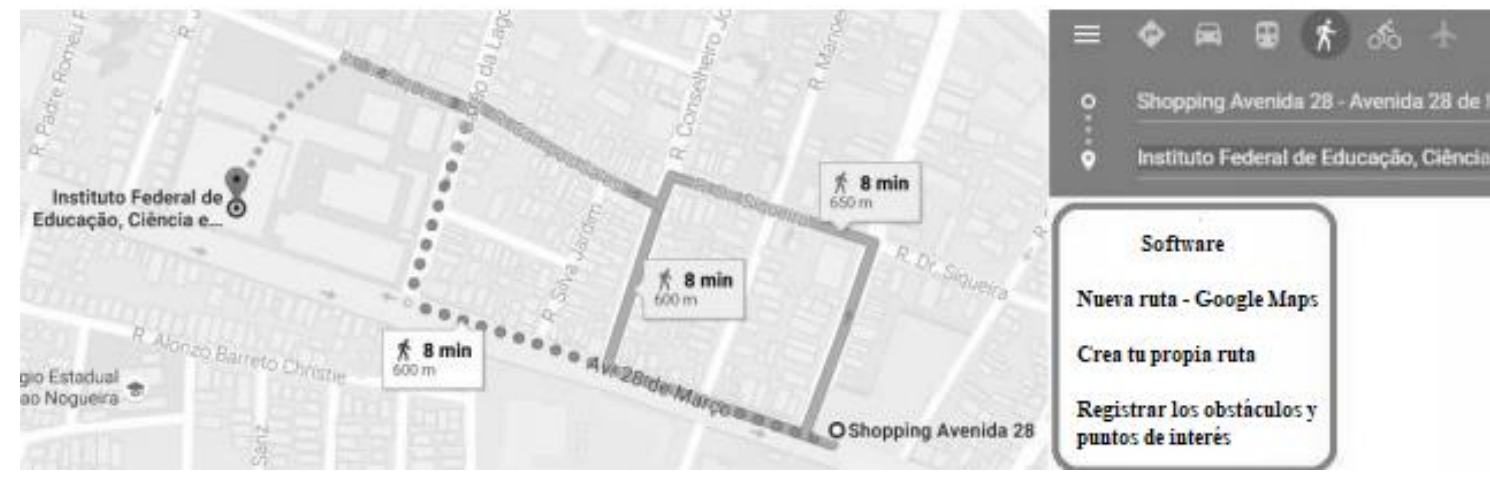

Figura 4. Uso de la aplicación para un entorno externo. 


\section{Características y ontología del sistema propuesto}

El modelo propone el desarrollo de una aplicación que tendrá las siguientes características:

- Crear y almacenar rutas desde Google Maps;

- Detectar obstáculos usando el bastón electrónico, y almacenarlos;

- Proporcionar la carga de mapas creados previamente para ayudar a la locomoción en ambientes internos;

- Desarrollar la mejor ruta en relación con la localización actual y la localización deseada;

- Almacenar las rutas más utilizadas;

- Guiar a las personas con discapacidad visual en entornos externos / internos a través del audio;

- Generar configuraciones personalizadas, donde el usuario puede configurar los audios y señales de sonido, seleccionando si quiere audio, señales de sonido o vibraciones en relación al bastón, ajustar su intensidad e incluso los modelos de voz y sonido deseados;

- Compartir la localización con otros, así como las rutas utilizadas;

- Identificación y lectura de QR-Code, permitiendo el acceso a lugares en entornos cerrados e incluso información adicional sobre ellos y objetos.

Garantizar la movilidad autónoma de personas con discapacidad visual interior del edificio no es una tarea sencilla. El sistema propuesto ayudará al usuario al informar el nombre del lugar y sugerir formas de llegar al destino previsto. Contiene sistemas similares, pero esta diferencia es importante en relación con los demás, ya que el modelo propuesto se basó en:

- Sistema abierto con licencias gratuitas de hardware y software;

- Mapas específicos para aumentar la accesibilidad en entornos cerrados;

- Creación de rutas personalizadas, marcando puntos de interés;

- Almacenamiento de obstáculos;

- Se pueden desarrollar salidas para navegación en entornos cerrados para cualquier entorno público de difícil locomoción para personas con necesidades visuales como: aeropuertos, universidades y centros comerciales;

- Acceso a la información en dos sectores de un entorno cerrado, mediante la identificación y lectura del QR-Code disponible en puertas y paneles informativos. Asimismo, acceso a información sobre productos en estanterías, obras de arte en exposiciones, etc. 
Para el desarrollo de aplicaciones, el almacenamiento de información seguirá el modelo de datos que representa el conjunto de objetos de dominio manejados por la aplicación y las relaciones entre ellos. Este modelo de datos se denomina ontología de dominio y se puede ver en la Figura 5. La ontología propuesta describe los tipos de Obstáculo, además de las relaciones, es decir, las formas en que los objetos se relacionan entre sí.

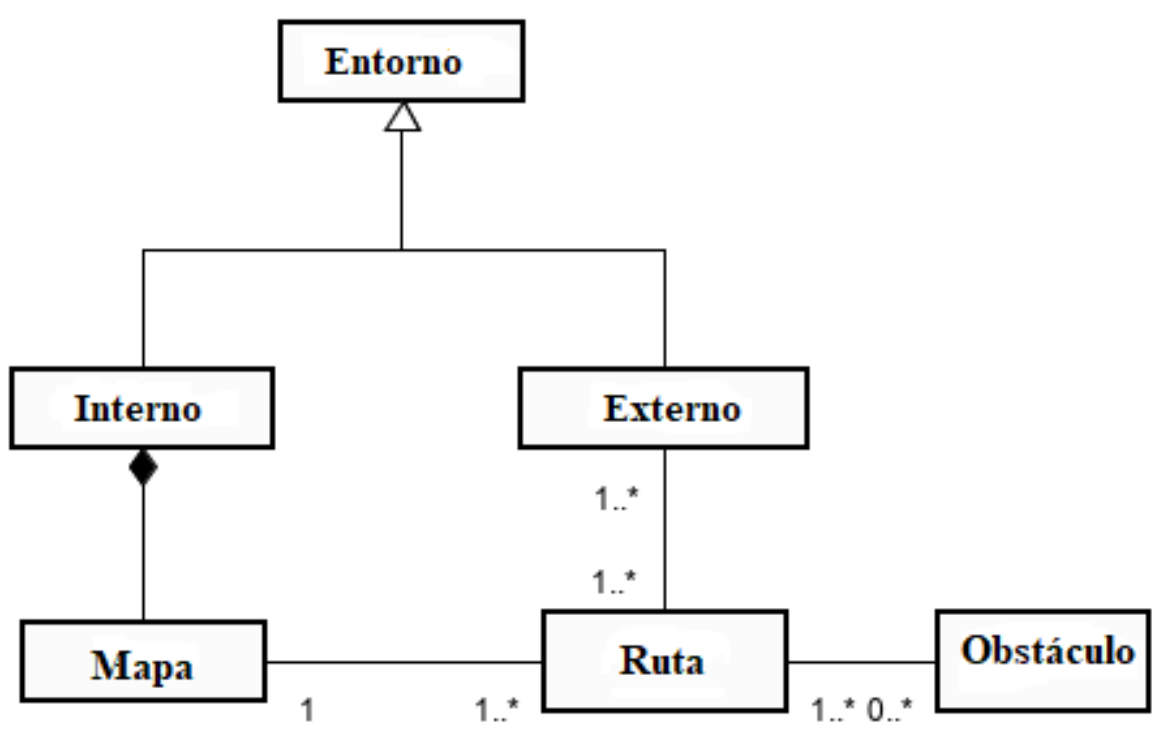

Figura 5. Ontología para el dominio de la aplicación.

En la Figura 5, la clase "Entorno" es una generalización de dos tipos de entorno: interno y externo. La clase "Interno" es una composición de mapas, donde la clase "Mapa" puede tener una o varias rutas, así como el entorno externo. Para la clase "Ruta", se pueden almacenar de ninguno a innumerables obstáculos. Para el entorno externo se utilizan mapas de Google Maps y el usuario puede almacenar una o varias rutas para cada uno de estos entornos. El entorno interno, se almacena el mapa de localización y el usuario puede almacenar una o varias rutas deseadas para cada uno dos entornos. Para cada ruta almacenada, el usuario también puede almacenar los obstáculos detectados por el bastón. Tanto el bastón electrónico como la aplicación serán desarrollados y probados en construcción a través de un proyecto piloto para promover la accesibilidad e inclusión social de estudiantes con discapacidad visual a través de la locomoción autónoma en las calles de la ciudad de Campos dos Goytacazes, en el estado de Río de Janeiro, y en los edificios y bloques del campus Campos Centro del Instituto Federal Fluminense (IFFluminense). Para esta etapa de validación se estableció una alianza con el Centro de 
Apoyo IFFluminense a Personas con Necesidades Educativas Especiales (NAPNEE). A través de la retroalimentación del equipo de invidentes que probará el equipo (bastón y aplicación) se recolectarán los resultados para medir la eficiencia y efectividad del proyecto.

\section{CONCLUSIONES}

Este trabajo propuso un modelo para un sistema autónomo de locomoción en entornos exteriores e interiores para personas con discapacidad visual, cuyo objetivo es facilitar la movilidad de las personas con discapacidad visual, permitiéndoles moverse de forma independiente en entornos exteriores e interiores, evitando el contacto con superficies y terceros, reduciendo el riesgo de contagio por Covid-19.

El sistema propuesto puede ser parte de un conjunto de medidas de protección contra Covid-19 establecido por IFFluminense y a partir de este proyecto piloto, el sistema puede ser utilizado por otras instituciones y gobiernos en apoyo a la Política Nacional de Movilidad, a través del Sistema Nacional de Movilidad Urbana. Contribuyendo de esta manera como facilitador de la movilidad de las personas con discapacidad visual y también para reducir el riesgo de contagio por Covid-19 de este grupo con un alto nivel de vulnerabilidad entre la población brasileña.

Mediante el uso del sistema propuesto se recolectarán indicadores efectivos de sus usuarios, así como se realizarán investigaciones para monitorear el tiempo de adaptación de las personas con discapacidad visual al nuevo modelo, la facilidad de manejo de la aplicación y el bastón electrónico. Para ello, se establecieron algunos indicadores: (i) tiempo para ubicar una determinada calle; (ii) tiempo para de localizar un cierto lugar situado en un establecimiento; (iii) usabilidad del bastón y la aplicación; (iv) efectividad del bastón en la detección de obstáculos; (v) efectividad del bastón en términos de detección de movimiento.

Como trabajo futuro, se sugiere realizar el proyecto piloto en las calles de la ciudad de Campos dos Goytacazes (entorno externo) y en las instalaciones de IFFluminense (entorno interno), para recolectar y analizar los indicadores obtenidos para una evaluación de la propuesta de sistema autónomo de modelo de locomoción. 


\section{REFERENCIAS BIBLIOGRÁFICAS}

Abdullah, S., Noor, N. M. y Ghazali, M. Z. (2014). Mobility recognition system for the visually impaired. IEEE 2nd International Symposium on Telecommunication Technologies (ISTT), 362-367, https://doi.org/10.1109/ISTT.2014.7238236

Barati, F. y Delavar, M. R. (2015). Design and Development of a Mobile Sensor Based the Blind Assistance Wayfinding System. The International Archives of Photogrammetry, Remote Sensing and Spatial Information Sciences, XL-1-W5 91-16, https://doi.org/10.5194/isprsarchives-XL-1-W5-91-2015

Barbosa, A. S. (2016). Mobilidade urbana para pessoas com deficiência no Brasil: um estudo em blogs. Brazilian Journal of Urban Management 8 (1) 142-154, https://doi.org/10.1590/2175-3369.008.001.AO03

Campos, A. C. (2020). Em tempos de COVID-19, deficientes visuais devem redobrar os cuidados. Rio de Janeiro: Agência do Brasil.

Castillo-Cara, M., Hauranga-Junco, E., Mondragón-Ruiz, G. A., Barbosa, L. O. y Antúnez, E. (2016). Ray: Smart Indoor/Outdoor Routes for the Blind Using Bluetooth 4.0 BLE. Procedia Computer Science, The 7th International Conference on Ambient Systems, Networks and Technologies (ANT 2016) / The 6th International Conference on Sustainable Energy Information Technology (SEIT2016) / Affiliated Workshops 83, 690-694, https://doi.org/10.1016/j.procs.2016.04.153

Cheraghi, S. A., Namboodiri, V. y Walker, L. (2017). GuideBeacon: Beacon-based indoor wayfinding for the blind, visually impaired, and disoriented. 2017 IEEE International Conference on Pervasive Computing and Communications, PerCom 2017, 121-130, https://doi.org/10.1109/PERCOM.2017.7917858

Coughlan, J. y Manduchi, R. (2009). Functional assessment of a camera phone-based wayfinding system operated by blind and visually impaired users. International Journal of Artificial Intelligence Tools 18 (3) 379-397, https://doi.org/10.1142/S0218213009000196

Flores, G. H., Manduchi, R. y Zenteno, E. D. (2014). Ariadne's thread: Robust turn detection for path back-tracing using the iPhone. 2014 Ubiquitous Positioning Indoor Navigation and Location Based Service, UPINLBS 2014 - Conference Proceedings, 133-140, https://doi.org/10.1109/UPINLBS.2014.7033720 
Huang, H., Lin, N. C., Barrett, L., Springer, D., Wang, H. C., Pomplun, M. y Yu, L. F. (2018). Automatic Optimization of Wayfinding Design. IEEE Transactions on Visualization and Computer Graphics 24 (9) 2516-2530, https://doi.org/10.1109/TVCG.2017.2761820

Instituto Brasileño de Geografía y Estadística [IBGE]. (2013). Pesquisa Nacional de Saúde. IBGE, Brasil. https://biblioteca.ibge.gov.br/visualizacao/livros/liv94522.pdf

Jafri, R. y Ali, S. A. (2014). A GPS-Based Personalized Pedestrian Route Recording Smartphone Application for the Blind. In: STEPHANIDIS, C. (Ed.). HCI International 2014 - Posters' Extended Abstracts. Communications in Computer and Information Science, 435 232-237. Springer, https://doi.org/10.1007/978-3319-07854-0_41

Jaind, D., Jain, A., Paul, R., Komarika, A. y Balakrishnan, M. (2013). A path-guided audio based indoor navigation system for persons with visual impairment. Proceedings of the 15th International ACM SIGACCESS Conference on Computers and Accessibility - ASSETS '13, 1-2, https://doi.org/10.1145/2513383.2513410

Koutny, R. y Miesenberger, K. (2015). Virtual Mobility Trainer for Visually Impaired People. Technology and Disability 26 (4) 211-219, https://doi.org/10.3233/TAD$\underline{140420}$

Legge, G. E., Beckmann, P. J., Tjan, B. S., Havey, G., Kramer, K., Rolkosky, D. y Rangarajan, A. (2013). Indoor Navigation by People with Visual Impairment Using a Digital Sign System. PLoS ONE 8 (10) 1-15, https://doi.org/10.1371/journal.pone.0076783

Li, B., Munoz, J. P., Rong, X., Chen, Q., Xiao, J., Tian, Y. y Yousuf, M. (2019). Visionbased Mobile Indoor Assistive Navigation Aid for Blind People. IEEE Transactions on Mobile Computing $18 \quad$ (3) 702-714, https://doi.org/10.1109/TMC.2018.2842751

Loeliger, E. y Stockman, T. (2013). Wayfinding without visual cues: Evaluation of an interactive audio map system. Interacting with Computers 26 (5) 403-416, https://doi.org/10.1093/iwc/iwt042 
Manduchi, R. (2012). Mobile Vision as Assistive Technology for the Blind: An Experimental Study. Computers Helping People With Special Needs 7383 (1) 916, https://doi.org/10.1007/978-3-642-31534-3_2

Manduchi, R., Kurniawan, S. y Bagherinia, H. (2010). Blind guidance using mobile computer vision: a usability study. ASSETS '10: Proceedings of the 12th international ACM SIGACCESS conference on Computers and accessibility, 241-242, https://doi.org/10.1145/1878803.1878851

Ministerio de la Mujer, la Familia y los Derechos Humanos [MMFDH]. (2020). Cartilha Informativa sobre os cuidados que devem ser tomados pelas pessoas com deficiência e doenças raras durante a pandemia do novo coronavírus (Covid-19). MMFDH, Brasil. https://sway.office.com/tDuFxzFRhn1s8GGi?ref=Link

Organización Mundial de la Salud [OMS]. (2019). World Report on Vision. OMS. https://www.who.int/publications/i/item/9789241516570

Rafian, P. y Legge, G. E. (2017). Remote Sighted Assistants for Indoor Location Sensing of Visually Impaired Pedestrians. ACM Transactions on Applied Perception 14 (3) 1-14, https://doi.org/10.1145/3047408

Tao, Y., Ding, L., Wang, S. y Ganz, A. (2017). Perfect Indoor Wayfinding for Blind and Visually Impaired Users: Navigation Instructions Algorithm and Validation Framework. Proceedings of the 3rd International Conference on Information and Communication Technologies for Ageing Well and e-Health, n. ICT4AWE, 143149, https://doi.org/10.5220/0006312001430149

Valentim, R. A. M, Cardoso, P. H., Souza, B. L., Morais, P. S. G., Morais, A. H. F., Silva, R. D., Cabral, J.V.F., y Lins, H. W. de C. (2016). Olho Biônico para Auxílio à Locomoção Autônoma de Deficientes Visuais. Revista Brasileira de Inovação Tecnológica em Saúde 6 (1) 35-43, https://doi.org/10.18816/r-bits.v6i1.7793

Vasconcelos, B. M., Teti, B. S., Figueira, A. M. A. y Gonçalves, L. M. S. (2020). Tecnologias assistivas destinadas à orientação espacial, identificação de obstáculos e guiamento de pessoas com deficiência visual. Gestão e Tecnologia de Projetos 15 (2) 52-68, https://doi.org/10.11606/gtp.v15i2.161697

Wang, S., Pan, H., Zhang, C. y Tian, Y. (2014). RGB-D image-based detection of stairs, pedestrian crosswalks and traffic signs. Journal of Visual Communication and Image Representation 25 (2) 263-272, https://doi.org/10.1016/j.jvcir.2013.11.005 
Zhang, H. y Ye, C. (2016). An Indoor Wayfinding System Based on Geometric Features Aided Graph SLAM for the Visually Impaired. IEEE Transactions on Neural Systems and Rehabilitation Engineering 25 (9) 1592-1604, https://doi.org/10.1109/TNSRE.2017.2682265

\section{PARA CITAR EL ARTÍCULO INDEXADO.}

Vasconcelos Silva, S., Pessanha Junior, E. A., \& Ferreira Nunes , C. (2021). Modelo de sistema de locomoción autónomo: una ayuda para las personas con discapacidad visual en la prevención de la Covid-19. Magazine De Las Ciencias: Revista De Investigación E Innovación, 6(2), 25-43. https://doi.org/10.33262/rmc.v6i2.1248 